\title{
Hoja de cálculo para la cuantificación del entrenamiento en piragüismo Spreadsheet for training quantification in canoeing
}

\author{
Fernando Alacid Cárceles \\ Universidad de Murcia (España)
}

\begin{abstract}
Resumen: El proceso de planificación y programación del entrenamiento deportivo es una de las actividades más difíciles que realizan los entrenadores, ya que conlleva una gran complejidad valorar la interacción de los diferentes tipos de cargas y contenidos de entrenamiento. Por otro lado, la cuantificación del entrenamiento realizado o planificado, en ocasiones, puede convertirse en una tarea aburrida y repetitiva, por lo que el uso de una herramienta informática de carácter genérico, como una hoja de cálculo, puede facilitar y ahorrar mucho tiempo al entrenador en este tipo de actividades, además de servir para obtener información de forma instantánea de lo planificado a lo largo de la temporada. Por todo ello, el objetivo de este artículo fue aportar una hoja de cálculo sencilla, gratuita y práctica para la planificación y cuantificación del entrenamiento deportivo, en esta ocasión adaptada al piragüismo, pero adaptable a otros deportes.
\end{abstract}

Palabras clave: Piragüismo, cuantificación del entrenamiento, planificación, hoja de cálculo.

Abstract: The planning and programming process in sport training is one of the most difficult activities made by coaches, because analysing the interaction between different types of loads and task volume is a very complex activity. On the other hand, the quantification of the training volumes can sometimes be boring and repetitive. In this case, the use of computer tools, like spreadsheets, can help coaches by saving a lot of time in these tasks. Moreover, the data plan is gathered throughout the season and displayed in real time. The aim of this paper is to contribute with a free, easy and useful spreadsheet for the planning and programming of sport training, in this case related to canoeing, but also useful for others sports.

Key words: Canoeing, training quantification, planning, spreadsheet.

\section{Introducción}

El uso de los programas informáticos en el ámbito de las Ciencias del Deporte, está dirigido, principalmente, hacia el análisis y medición del rendimiento deportivo como herramientas para manejar el instrumental utilizado en estas valoraciones, siendo escasas las aportaciones como ayuda al entrenador en la programación y planificación del entrenamiento (Morante, 2000).

Para Morante (2000) el software desarrollado para las Ciencias del Deporte, se puede clasificar en tres grandes áreas:

- Área Fisiológica: aplicaciones para la medición de diversas variables como el consumo de oxígeno, la frecuencia cardiaca, la fuerza, aspectos antropométricos y de composición corporal, análisis electromiográfico, etc.

- Área Biomecánica: principalmente en la valoración de aspectos relacionados con la técnica deportiva como la digitalización y análisis cinemático, el uso de plataformas de fuerza, test aerodinámicos e hidrodinámicos, etc.

- Área Psicológica: en la valoración del tiempo de reacción, atención selectiva, biofeedback, test de coordinación motriz, y técnicas de visualización e imaginación mental, etc.

De manera general, este tipo de software suele estar diseñado para su uso específico hacia una determinada aplicación, limitando su utilización a los compradores de la aplicación o del instrumental para la cual está diseñada. El uso de programas informáticos de aplicación genérica (hojas de cálculo, procesador de textos, bases de datos, etc.), como los desarrollados por Microsoft Office u OpenOffice, facilita la difusión e intercambio de las aplicaciones o recursos desarrollados entre un alto número de entrenadores y deportistas, así como su total adaptación a las preferencias del usuario.

Las herramientas para el control y la cuantificación del entrenamiento no son la clave de una planificación exitosa, pero facilitan el proceso de elaboración, corrección y ajuste de las programaciones realizadas. La cuantificación de la carga facilita el aprendizaje sobre las

Fecha recepción: 25-04-08 - Fecha envío revisores: 25-04-08 - Fecha de aceptación: 08-05-08 Correspondencia: Fernando Alacid Cárceles

Calle Argentina, $s / n$.

30720 - Santiago de la Ribera - Murcia (España)

E-mail: fernando.alacid@um.es planificaciones anteriores y aporta una valiosa información sobre la vida deportiva de nuestros deportistas (Alacid \& Torres, 2006).

La gran cantidad de información que hay que manejar durante el proceso de planificación y entrenamiento (volumen, intensidad, diferentes períodos, etc.), y el tiempo que se emplea en tratarla de forma manual, en muchas ocasiones supone una tarea aburrida y repetitiva, hace más que necesario el uso de este tipo de aplicaciones para gestionar la información (Rivas, 2003).

Existen en el mercado algunos ejemplos, principalmente centrados en deportes individuales de carácter cíclico. Destacan los citados por Morante (2000): WinTrainer (Rogers, 1995), On Your Mark (Full Sail Software, 1995), Planificación y control del entrenamiento del ciclistal nadador (García, 1996a, 1996b) y del corredor de resistencia (García, 1996c), Polar Sports Diary SW (Polar Electro Oy, 1997) y PC Coach Logbook, PC Coach-Professionaly PC Pro Training Series (Biometrics Inc, 1997a, 1997b, 1997c) entre otros; y el software para la planificación y programación del entrenamiento en natación (Navarro, Rivas, \& Muñiz, 2001; Rivas, 2003).

El ejemplo que se expone a continuación está basado en la hoja de cálculo Excel de Microsoft, consiste en sumar los volúmenes de entrenamiento mientras se realizan los microciclos, obteniendo automáticamente la cuantificación de los diferentes períodos de entrenamiento de forma inmediata. Se ha realizado una adaptación a los contenidos más utilizados en piragüismo, diferenciando entre en el entrenamiento en agua (Sánchez, 1993) y en seco (Sánchez-Mota, 1993). La cuantificación del entrenamiento en agua se realiza en kilómetros y horas, mientras que el entrenamiento en seco, únicamente se cuantifica en horas. Se da la posibilidad de cambiar los contenidos expuestos para adaptar la hoja de cálculo a cualquier otra especialidad deportiva que cuantifique las cargas de una forma similar.

Algunas de las características de esta hoja de cálculo son, en primer lugar el uso únicamente de microciclos de una semana de duración, ya que de esta forma, se facilita la cuantificación de las cargas. En segundo lugar, se cuenta con un apartado para el almacenamiento de los entrenamientos realizados, facilitando la creación posterior de los microciclos y el intercambio de entrenamientos entre técnicos.

Por todo ello, el objetivo de este artículo fue, en primer lugar, difundir una herramienta gratuita, sencilla y práctica para la planificación y cuantificación del entrenamiento deportivo y en segundo lugar, explicar el funcionamiento y las posibles adaptaciones de esta hoja de cálculo a otros deportes. 


\section{La hoja de cálculo}

El archivo Entto.xls, posee las siguientes hojas:

- PLANIF

ENTTOS

- M1 - M52

SECO SEM

- AGUA SEM

- SECOAC

- AGUAAC

Para facilitar la explicación de las diferentes hojas y hacer referencia a las celdas que se deben rellenar, a lo largo del texto cuando se indique una celda se hará entre paréntesis colocando en primer lugar la letra de la columna y en segundo lugar el número de la fila. De este modo, para referirse a la primera celda de una hoja de cálculo se empleará(A1). Por otro lado, para indicar un conjunto de celdas, se utilizará la primera y la última de ellas separadas por dos puntos. Así, al indicar las cuatro celdas (A1), (A2), (B1) y (B2), únicamente se utilizarán la primera y laúltima de las señaladas (A1:B2).

\section{La hoja PLANIF}

Recoge toda la información de la temporada, su planificación, organización y cuantificación general. Destacan varios apartados dentro de lamisma:

1. Información general de la planificación: en la que se debe cumplimentar el año de la temporada (D2), el nombre del equipo (K2) y del entrenador (W2). Estos datos aparecerán en todos los microciclos.

2. Competiciones: se indicarán los meses del año (fila4), el domingo de cada semana de la planificación (fila 6), los objetivos principales (fila 7) y secundarios de la temporada (fila 8) y la denominación de las pruebas (fila 9).

Se recomienda el uso de combinar celdas (señalar las celdas'! botón derecho del ratón '! formato de celdas '! alineación '! combinar celdas) para indicar la extensión de los meses en la fila 4.

3. Periodización: en las filas 10 a 12 se indican las unidades de planificación y en la 13 los objetivos de los mesociclos de la temporada, en ella, se recomienda el uso de las abreviaturas de los contenidos del entrenamiento. Del mismo modo que en el apartado anterior, el uso de combinar celdas mejora la presentación de esta parte de la planificación.
En la fila 14 se hace referencia a cada uno de los microciclos de la temporada (hojas M1 - M52), a los que podemos acceder pulsando sobre su número mediante el uso de un hipervínculo.

Las filas 15 a 22 corresponden a una representación de la distribución de la carga del 30 al $100 \%$ de un máximo teórico. El uso de un color para rellenar las celdas es la forma más sencilla de completar este apartado, siendo de cualquier modo, totalmente modificable según las necesidades del usuario.

Las últimas tres filas se utilizan para indicar los microciclos en los que se pretende realizar un control médico (fila 23), de estado de forma (fila 24) o existe algún tipo de evento importante que señalar en la planificación (fila 25) como un desplazamiento largo, una concentración, etc.

En figura 1 muestra un ejemplo de los tres primeros apartado de la hoja PLANIF.

4. Distribución de los contenidos: este apartado ha sido dividido en dos bloques de contenidos bien diferenciados: sesiones semanales de entrenamiento en agua y sesiones semanales de entrenamiento en seco. Además, en las celdas (D26:D36) y (D38:D47), sombreadas en amarillo, se establecen los contenidos de entrenamiento sobre los que se realizará la cuantificación. En este caso están puestos como ejemplo los más utilizados en piragüismo de aguas tranquilas(Sánchez, 1993; SánchezMota, 1993) (figura 2).

Con el fin de poder utilizar esta herramienta en otros deportes o establecer otros contenidos de entrenamiento diferentes en piragüismo, la realización de cualquier cambio en la denominación de los contenidos en las celdas indicadas anteriormente, conlleva la variación en el resto de hojas utilizadas en las que se representen estos contenidos. Es decir, si se pretende cuantificar el entrenamiento de esquí de fondo y además no se posee una balsa de entrenamiento especial, en la celda (D47) se modificaría $B A L$ por $S K I$, haciéndose permanente el cambio en el resto de las hojas.

En las celdas(E26:BD36) y (E38:BD47), se indicarán las sesiones semanales por contenidos de agua o en seco, se han establecido diferentes formatos en las celdas en función del número que se escriba, con el fin de facilitar la identificación de los momentos de la temporada en los que se pretende desarrollar de una forma más intensa unos u otros contenidos.

5. Cuantificación: se representan los kilómetros realizados en agua y las horas de entrenamiento en seco para cada uno de los contenidos de

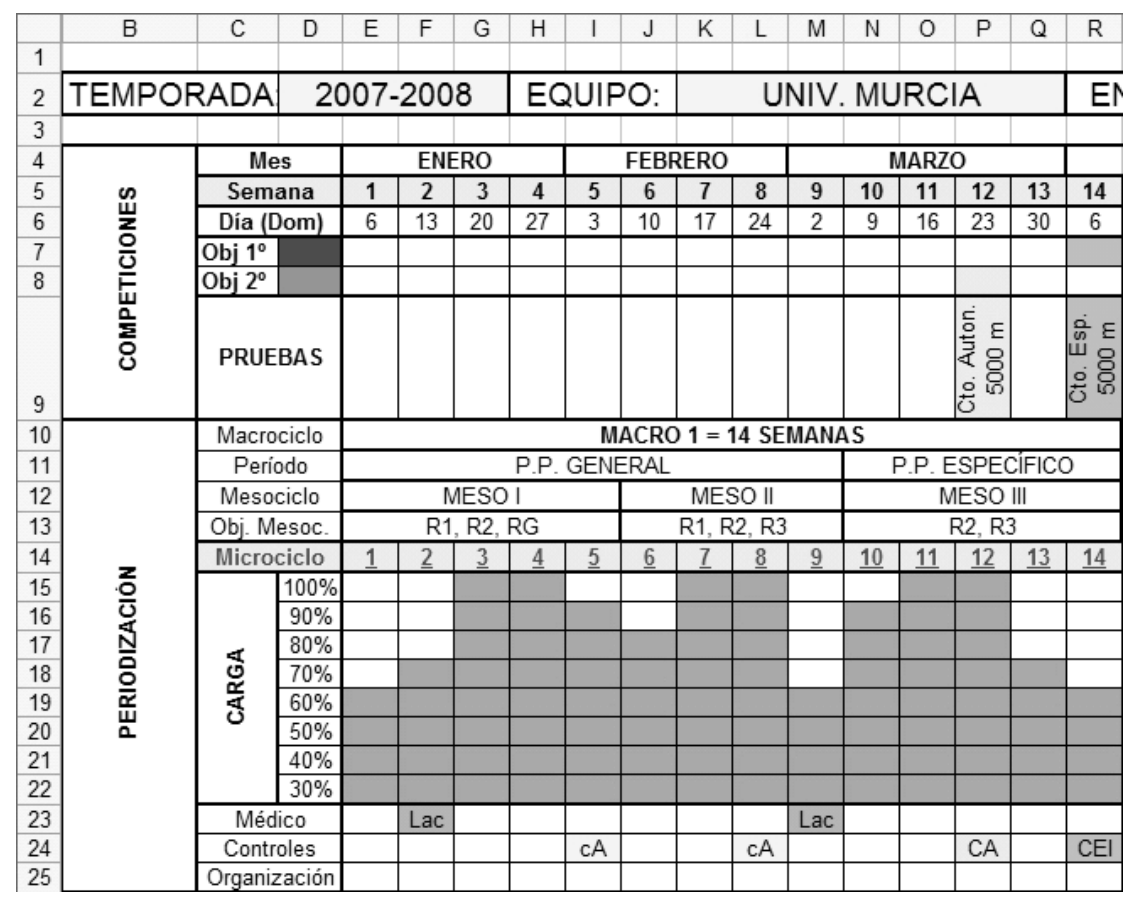

Figura 1.Información general de la planificación 


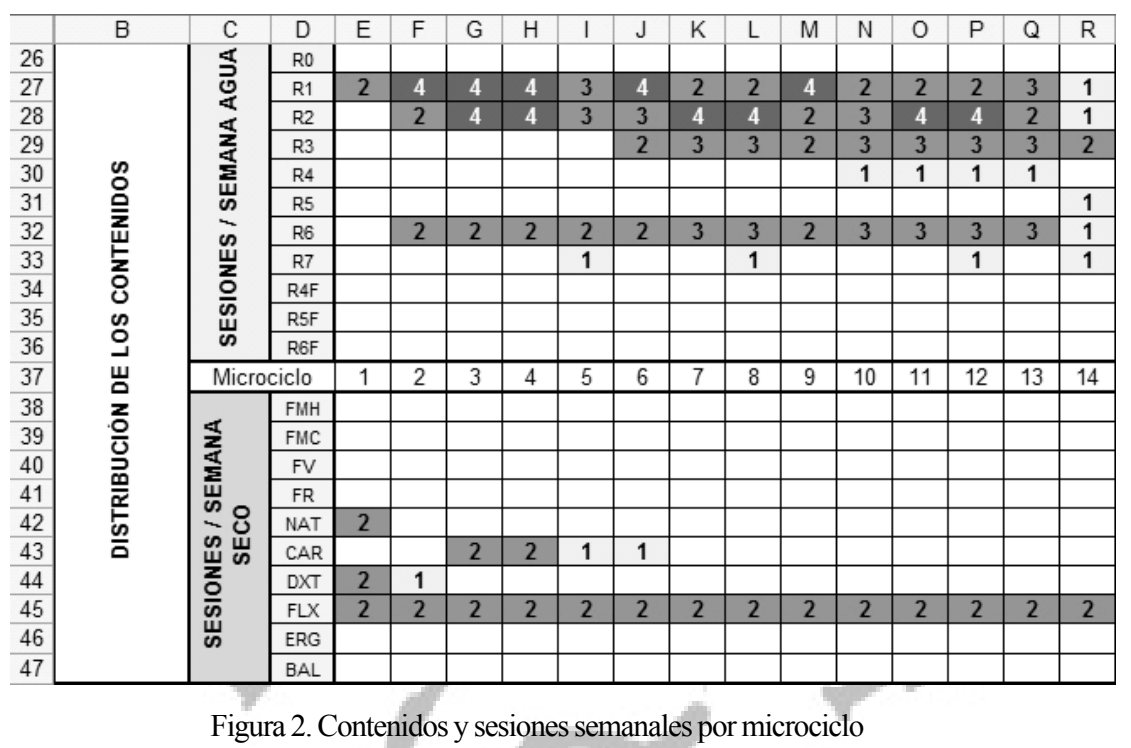

entrenamiento y microciclo. Esta cuantificación se realiza automáticamente conforme se vayan rellenado las hojas correspondientes a los microciclos, por lo que a partir de este momento, no es necesario rellenar nada más en la hoja PLANIF.

En las celdas (E71:BD73) aparecerán el total de kilómetros y horas (en seco y totales) por cada microciclo, mientras que en (BE49:BE70) lo harán para toda la temporada por contenido. En las celdas (BE71:BE73) se representan el sumatorio total de kilómetros y horas de la temporada.

En la parte inferior de la hoja, figura la leyenda de los contenidos de entrenamiento utilizados como ejemplo.

También se presentan otras dos cuantificaciones, pero esta vez con los microciclos colocados en una columna en lugar de una fila. En la primera de ellas, se indican los kilómetros y horas por microciclo y en la segunda se van sumando microciclo a microciclo, por lo que se denomina cuantificación semanal acumulada.

Para terminar, debajo de las cuantificaciones, encontramos dos gráficos que representan el volumen total en kilómetros y horas microciclo a microciclo y de forma acumulativa. En ellos, no se hace ningún tipo de diferenciación por contenidos.

\section{La hoja ENTTOS}

Está destinada a la recopilación y clasificación de los entrenamientos realizados (figura 3). En la fila 3 aparecen los contenidos que han sido establecidos en las celdas (D26:D47) de la hoja PLANIF.
Para la realización de un entrenamiento, debemos rellenar una fila completa desde la columna A a la Z (siempre a partir de la fila 4).

La columna A, hace referencia al tipo de entrenamiento, por lo que indicaremos la abreviatura del contenido a desarrollar.

En la columna $C$ se indicará la descripción del entrenamiento, por lo que debe aparecer al menos el tiempo o kilómetros a realizar, el contenido y la intensidad cuando éste último no sea indicador de la misma. Esta descripción es la que posteriormente aparecerá en la hoja de entrenamiento que será publicada, por lo que es interesante especificar todo lo que sea necesario para facilitar la comprensión del mismo por el deportista.

En las columnas D a X, pondremos el volumen en kilómetros si se trata de contenidos de entrenamiento en agua u horas si son de entrenamiento en seco, siempre debajo del contenido que se desarrolle.

La columna $Y$ presenta el sumatorio de kilómetros realizados en agua, mientras que en la columna $Z$, debemos escribir el número de horas de entrenamiento, ya sean en seco o en agua. Para facilitar el paso de minutos a horas, en la parte superior de la hoja (L1) se pueden introducir los minutos que aparecerán en horas en la celda (R1).

A veces puede resultar complicado conocer la correspondencia entre el tiempo y los kilómetros de agua realizados, ya que es un dato que no se puede generalizar entre las diferentes categorías existentes. La experiencia y observación es la principal ayuda en la obtención de esta correspondencia.

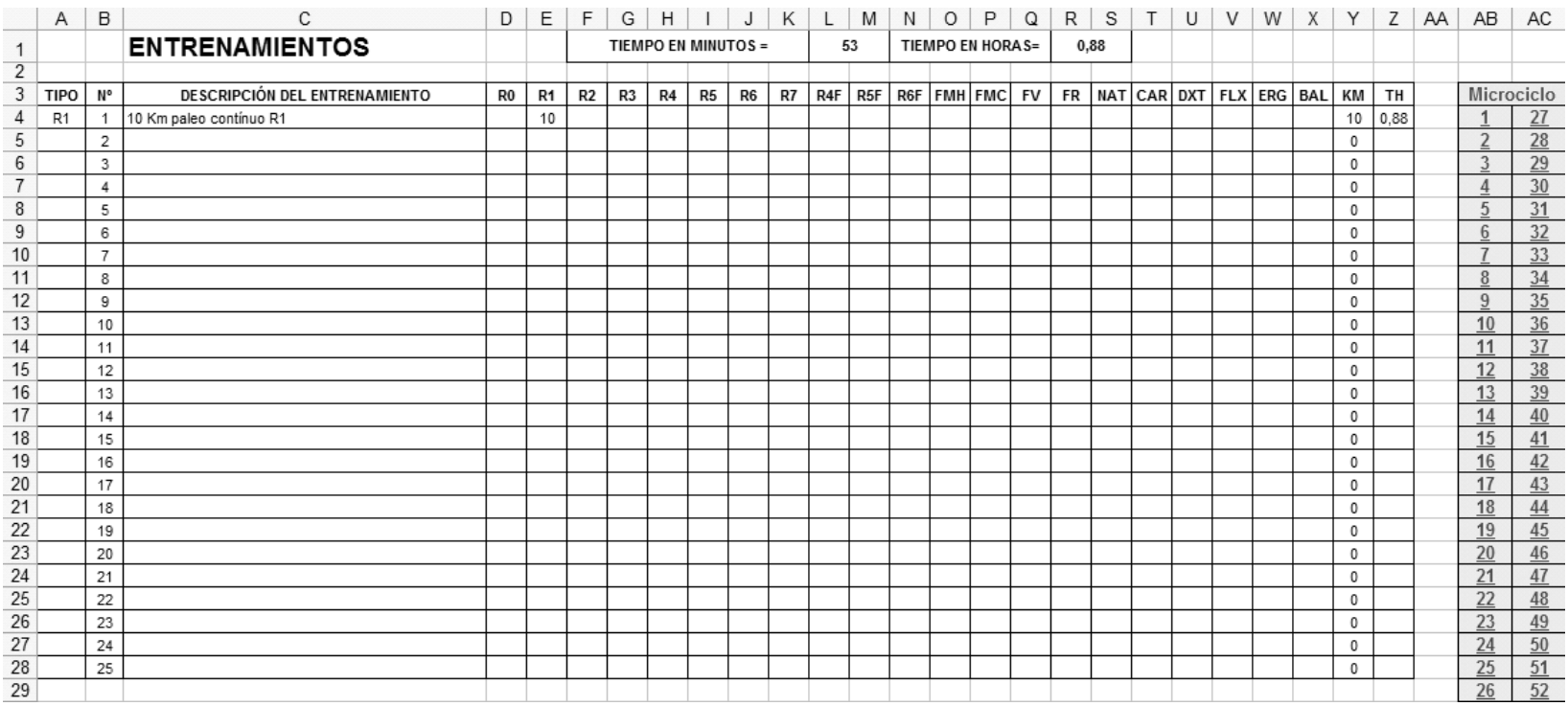

Figura 3. La hoja ENTTOS 


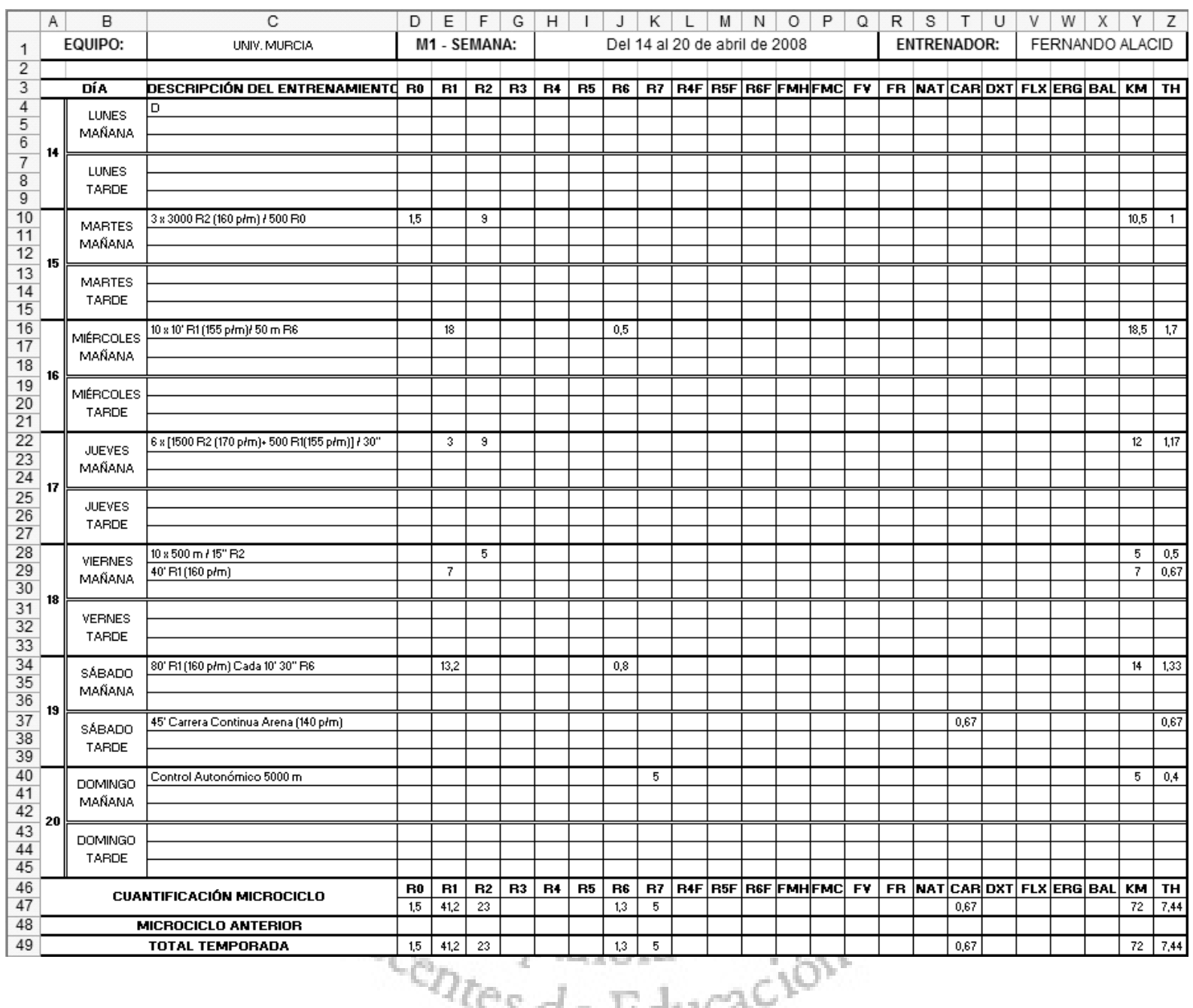

Figura 4. Realización de los microciclos en las hojas M1-M52.

Cuando se disponga de un número alto de entrenamientos almacenados, podremos ordenarlos mediante el uso del comando ordenar (datos '! ordenar). Previamente se debe seleccionar la fila completa de los entrenamientos, desde la fila A a la Z, ya que de otro modo, se mezclarían unos entrenamientos con otros. Se recomienda ordenarlos en primer lugar por la columna $T I P O$ y después por la columna $T H$, de esta forma, se dispondrá de los entrenamientos de un mismo contenido ordenados de mayor a menor volumen.

En las columnas AB y AC, se han dispuesto hipervínculos a cada uno de los microciclos para facilitar el paso de la hoja de entrenamientos a la de los microciclos.

\section{Las hojas M1 - M52}

En estas hojas se realizarán los entrenamientos cuya cuantificación aparecerá en la hoja PLANIF y en las hojas de gráficos.

La hoja se estructura en 2 partes bien diferenciadas, de la columna A hasta la Z se realizan los entrenamientos (figura 4 ) y de la columna $\mathrm{AB}$ a la $\mathrm{AX}$ se exponen de una forma más visual y se acompañan con un gráfico que representa la evolución del volumen por días de la semana (figura5).

En la ceda $(\mathrm{H1})$ se escribe el período de tiempo que abarca la semana de entrenamiento, por ejemplo: «Del 14 al 20 de abril de 2008», mientras que en las celdas (A4:A45) se colocan los días de la semana: «14, 15, 16...20».

Para realizar los entrenamientos, rellenaremos las celdas (C4:Z45) del mismo modo que lo hacíamos en la hoja de ENTTOS. Lo más indicado es copiar la fila correspondiente en la hoja ENTTOS y pegarla en la del microciclo, y si se quiere mantener el formato de la hoja, es conveniente utilizar el comando pegado especial de valores (botón derecho'! pegado especial '! valores '! aceptar).

La hoja de los microciclos está diseñada para un máximo de dos sesiones diarias y tres contenidos por sesión. Si se quisiera poner más sesiones o contenidos, habría que combinarlos en una misma fila.

En la fila 47 se muestra un sumatorio por contenidos de entrenamiento del microciclo, en la 48 se indica lo realizado en la temporada hasta el momento y en la 49 lo que se llevaría tras sumar lo realizado en el microciclo actual.

En el resto de la hoja todos los datos aparecen automáticamente, pudiendo cambiar el tamaño de la fuente para adaptar la longitud de la descripción del entrenamiento al tamaño de la celda donde se expone.

En las celdas (B51) y (B53), se han realizado hipervínculos a las hojas PLANIF y ENTTOS respectivamente, para facilitar la movilidad entre hojas.

\section{Las hojas SECO SEM, AGUASEM, SECOAC,AGUAAC}

En estas hojas se representa gráficamente el volumen semanal por contenidos por microciclos y de forma acumulativa a lo largo de la temporada. Todas estas representaciones se realizan de forma automática conforme se van completando los microciclos de la temporada.

A modo de conclusión, esta hoja de cálculo aporta una herramienta de libre distribución, modificable y por tanto adaptable a las necesidades de los técnicos y deportistas y con un sencillo sistema de almacenamiento de entrenamientos y composición de microciclos. 


\section{Referencias}

Alacid, F., \& Torres, G. (2006). Herramientas para el control del entrenamiento deportivo en la iniciación deportiva. En C. Villaverde (Ed.), XVIII Edición de los cursos de verano de la Universidad de Granada en Ceuta (pp. 145-155). Ceuta: Instituto de Estudios Ceutíes.

Biometrics Inc. (1997a). PC Coach-Professional(Version2). Champaign, Illinois: Human Kinetics.

Biometrics Inc. (1997b). PC Coach Logbook. Champaign, Illinois: Human Kinetics.

Biometrics Inc. (1997c). PC Pro Training Series. Champaign, Illinois: Human Kinetics.

Full Sail Software. (1995). On Your Mark (Version 1.1). Fort Worth, Texas: Full Sail Software.

García, M. (1996a). Planificación y control del entrenamiento de la resistencia del ciclista. Madrid: Gymnos.

García, M. (1996b). Planificación y control del entrenamiento de la resistencia del nadador. Madrid: Gymnos.

García, M. (1996c). Planificación y control del entrenamiento del corredor de resistencia. Madrid: Gymnos.

Morante, J. C. (2000). Elaboración y validación de un programa informático interactivo de apoyo al entrenamiento deportivo. Tesis Doctoral no publicada. Universidad de León, León.

Navarro, F., Rivas, A., \& Muñiz, D. (2001). AETN - SPyCE (Version 1.0). Madrid: Gymnos.

Polar Electro Oy. (1997). Polar Sports Diary SW (Version 1.0). Kempele: Polar Electro Oy.

Rivas, A. (2003). Computer Science for Planning, Programming and Balance Process in Sport Coaching. International Journal of Computer Science in Sport, 2(1), 93-101.
Rogers, B. (1995). WinTrainer. Madrid: SanoCare Human Systems. Sánchez-Mota, J. (1993). Acondicionamiento físico. En J. L. Sánchez (Ed.), Piragüismo (II) (pp. 17-153). Madrid: COE.

Sánchez, J. L. (1993). Entrenamiento en agua. En J. L. Sánchez (Ed.), Piragüismo (II) (pp. 153-259). Madrid: COE.

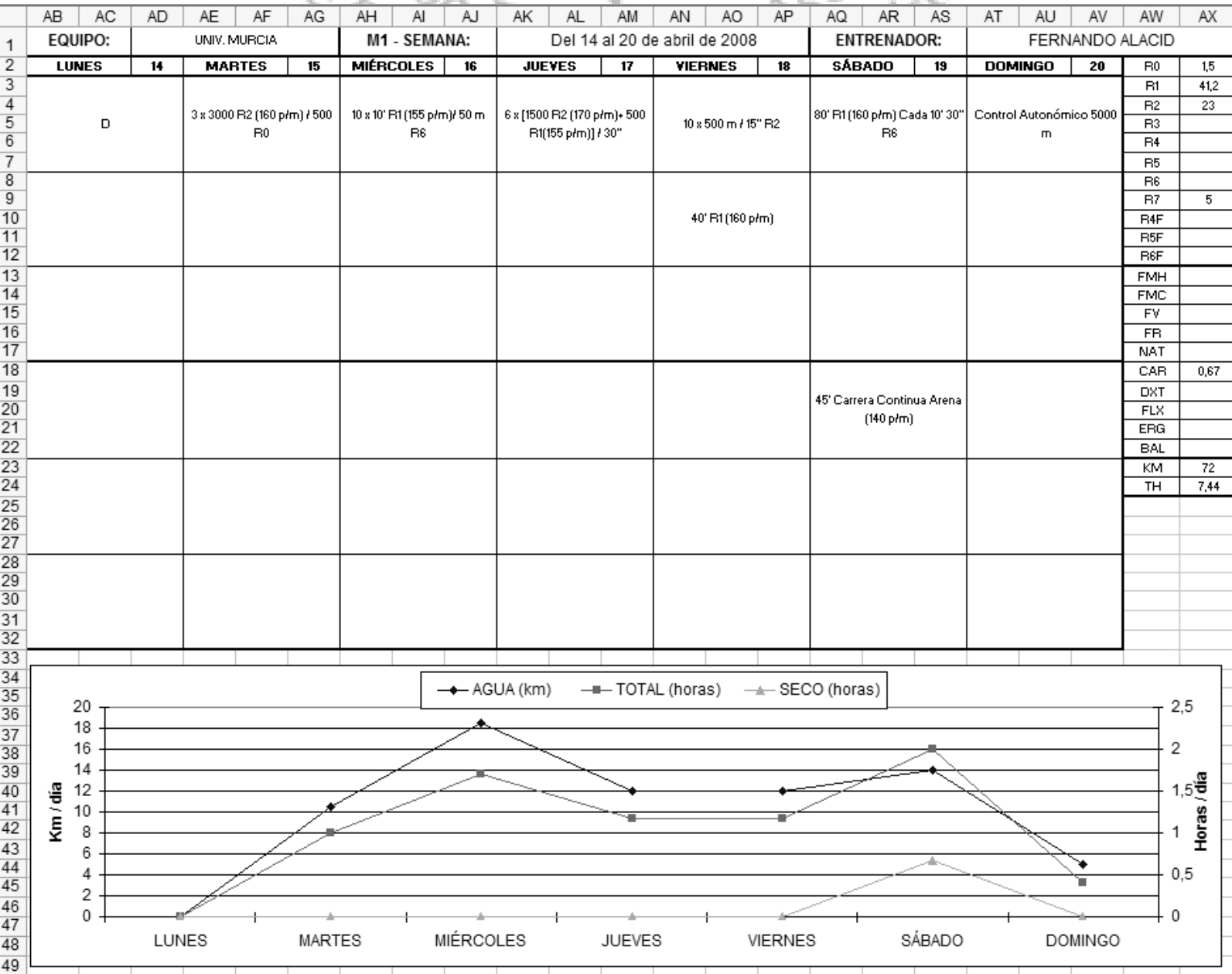

Figura 5. Exposición de los microciclos en las hojas M1-M52 